\title{
ANÁLISIS DE LA FRAGILIDAD PARA IDENTIFICAR SWEET SPOTS EN FORMACIONES DE SHALE GAS
}

\author{
Leonardo Fernández Rojas. ${ }^{1 *}$, Yair Andrés Quintero Peña² ${ }^{2}$ Zuly Himelda Calderón Carrillo ${ }^{3}$ \\ *A quien debe dirigirse la correspondencia
}

\begin{abstract}
RESUMEN
El objetivo de este trabajo es analizar el impacto del índice de fragilidad en el fracturamiento hidráulico de formaciones de shale gas a nivel mundial y definir parámetros para identificar zonas de interés o sweet spots fracturables en shale plays prospectos.
\end{abstract}

Se realizó una revisión bibliográfica de las propiedades petrofísicas y mecánicas que exhiben diversas formaciones de shale gas en países como Estados Unidos, China, Holanda, Noruega, Dinamarca y Suecia. Una vez estructurada la base de datos, se calcularon índices de fragilidad utilizando diferentes métodos teniendo en cuenta la composición mineralógica y los módulos elásticos estáticos y dinámicos de cada formación de shale estudiada.

El cálculo de índices de fragilidad en profundidad y su relación con el contenido orgánico total permitió identificar posibles sweet spots para las formaciones de shale gas de mayor prospección. Adicionalmente, el análisis de la relación entre el índice de fragilidad y el comportamiento mecánico de dichas formaciones permitió determinar que fluido de fractura es el apropiado para utilizar en caso de realizar un fracturamiento hidráulico en la zona de interés.

Palabras clave: Shale gas, fracturamiento hidráulico, índice de fragilidad, módulos elásticos, mineralogía, sweet spot.

\section{BRITTLENESS ANALYSIS TO IDENTIFY SWEET SPOTS IN SHALE GAS FORMATIONS}

\section{ABSTRAC}

The purpose of this paper is to analyze the impact of the Brittleness index in hydraulic fracturing on shale gas formations worldwide, and thereby define parameters to identify fraccable feasibly sweet spots in prospects shale plays.

A literature review was made so as to gather the largest amount of petrophysical and mechanical data from shale gas formations from countries all over the world such as United States, China, Holland, Norway, Denmark and Sweden. Once available the data, different methods were used to calculate the brittleness index of each study case considering the effect of the mineralogical composition and elastic moduli (static and dynamic).

The relation between the brittleness index at depth and the total organic carbon allowed to identify possible sweet spots within each prospect formation. Additionally, the analysis of brittleness index and mechanical behavior of those formations permitted to stablish which fracture fluid should be used in case of a hydraulic fracturing stimulation at the sweet spot.

Keywords: shale gas, hydraulic fracturing, brittleness index, elastic moduli, mineralogy, sweet spot

1. Grupo de Investigación de Estabilidad de Pozo, GIEP. Universidad Industrial de Santander, Bucaramanga, Colombia. leonardo.fernandez1@correo.uis.edu.co

2. Instituto Colombiano del Petróleo, ICP, Piedecuesta, Colombia. yair.quintero@ecopetrol.com.co

3. Grupo de Investigación de Estabilidad de Pozo, GIEP. Universidad Industrial de Santander, Bucaramanga, Colombia.calderon@uis.edu.co 


\section{INTRODUCCIÓN}

La producción shale gas mediante técnicas como la perforación horizontal y el fracturamiento hidráulico ha permitido a países desarrollados como Estados Unidos, encontrar una solución de mediano y largo plazo a la crisis energética actual [2]. Estas formaciones de shale poseen características únicas de alta heterogeneidad y permeabilidades ultra bajas, lo que dificulta el flujo de fluidos a través del espacio poroso de la roca, sin embargo son rocas que pueden generar grandes cantidades de gas y petróleo por su alto contenido de carbono orgánico total (TOC) [13].

El fracturamiento hidráulico es una técnica de estimulación basada en la inyección de fluidos a grandes tasas de bombeo hacia el pozo, con el fin de generar fracturas en la cara del pozo y sus vecindades, de tal manera que aumente la permeabilidad de la formación y por lo tanto el flujo de fluidos hacia el pozo. No obstante, dichas fracturas no permanecen abiertas por tiempo indefinido ya que los esfuerzos in situ ejercen sobre ellas cierta presión de cierre lo que implica una reducción gradual de la permeabilidad. Es por esta razón que junto al fluido de fractura se inyecta material propante, el cual tiene como función mantener abierta la fractura y soportar los esfuerzos in situ que actúan sobre la misma.

\section{MÓDULOS ELÁSTICOS Y FRACTURAMIENTO HIDRÁULICO}

Uno de los factores claves en un fracturamiento hidráulico exitoso es el comportamiento mecánico de la roca, quien determina la capacidad de la roca de sufrir una deformación o de fallar bajo esfuerzos, i.e. si la roca se puede fracturar o no. Existen diferentes constantes elásticas para describir el comportamiento mecánico de la roca (e.g. módulo de cizalla, módulo volumétrico, etc.), $\mathrm{y}$ todos describen, de una $\mathrm{u}$ otra forma, una relación entre esfuerzo y deformación, sin embargo en términos de fracturamiento hidráulico los módulos más importantes son el módulo de Young (E) y la relación de Poisson (v) [25].

El módulo de Young se define como la relación entre el esfuerzo axial y la deformación axial de la roca (Ecuación 1) y describe que tanto se deforma la roca a medida que aumenta el esfuerzo axial sobre ella. Es un factor determinante a tener en cuenta durante la planeación de un fracturamiento hidráulico en un intervalo de interés, ya que a mayor módulo de Young se garantiza mayor longitud y mantenimiento de la fractura [24] [25].

$$
E=\frac{\partial \sigma a x i a l}{\partial \text { saxial }}
$$

Dónde:

$E$ Módulo de Young [psi]

axial $=$ Esfuerzo axial [psi]

Eaxial $=$ Deformación axial [adimensional $]$

En la Tabla 1 se muestran valores comunes de módulo de Young para diferentes materiales.

Tabla 1. Módulo de Young para diferentes materiales

\begin{tabular}{|cc|}
\hline Material & Módulo de Young, psi \\
\hline Acero & $30.5 \mathrm{E}+06$ \\
\hline Aluminio & $10 \mathrm{E}+06$ \\
\hline Vidrio & $8.7 \mathrm{E}+06$ \\
\hline Concreto & $4.3 \mathrm{E}+06$ \\
\hline Plomo & $2.5 \mathrm{E}+06$ \\
\hline
\end{tabular}

Fuente: Howard \& Angus, 2006 [15].

Análogamente, se puede observar que rocas con un módulo de Young mayor a $10 \mathrm{E}+06$ psi se pueden comportar como el acero, el cual exhibe una deformación casi nula antes de fallar bajo esfuerzo. Por otro lado, rocas con módulos de Young menores a $3 \mathrm{E}+06$ psi se pueden comportar como el plomo, el cual es un material maleable y sufre un alto grado de deformación antes de fallar bajo esfuerzo.

La relación de Poisson se define como la relación entre la deformación radial y la deformación longitudinal de una roca sometida a un esfuerzo axial (Ecuación 2) y este describe que tendencia de deformación tiene la roca, bien sea expansión lateral o contracción axial. Dicho parámetro permite identificar zonas donde la roca se fracturará con mayor facilidad bajo esfuerzos, i.e. a menor relación de Poisson, la roca tendrá una mayor tendencia a fracturarse [24][29].

$$
v=\frac{\text { deradial }}{\text { delongitudinal }}
$$

Donde:

$v=$ Relación de Poisson [adimensional]

Eradial $=$ Deformación radial

[adimensional]

Elongitudinal $=$ Deformación longitudinal

[adimensional] 
La Tabla 2 presenta valores comunes de relación de Poisson para diferentes materiales.

Tabla 2. Relación de Poisson para diferentes materiales.

\begin{tabular}{|cc|}
\hline Material & Relación de Poisson \\
\hline Plomo & 0.43 \\
\hline Aluminio & 0.33 \\
\hline Acero & 0.29 \\
\hline Vidrio & 0.22 \\
\hline Concreto & 0.2 \\
\hline
\end{tabular}

Fuente: Dooley, 2010 [10].

$\mathrm{Si}$ se comparan los valores, rocas con relaciones de Poisson entre 0.2 y 0.3 van a presentar un comportamiento similar al vidrio o al acero, los cuales presentan muy poca deformación bajo esfuerzos, lo que implica mayor facilidad para fracturarse. Rocas con relaciones de Poisson mayores a 0.4 indican un alto grado de deformación radial bajo esfuerzos, lo que implica un cambio de forma significativo en la roca, este comportamiento dúctil es común en materiales maleables como el plomo.

La Figura 1 ilustra los valores de módulo de Young y relación de Poisson estáticos y dinámicos para diferentes formaciones de shale gas a nivel mundial [1][11][12][17][23] [24][27][28][35][39].
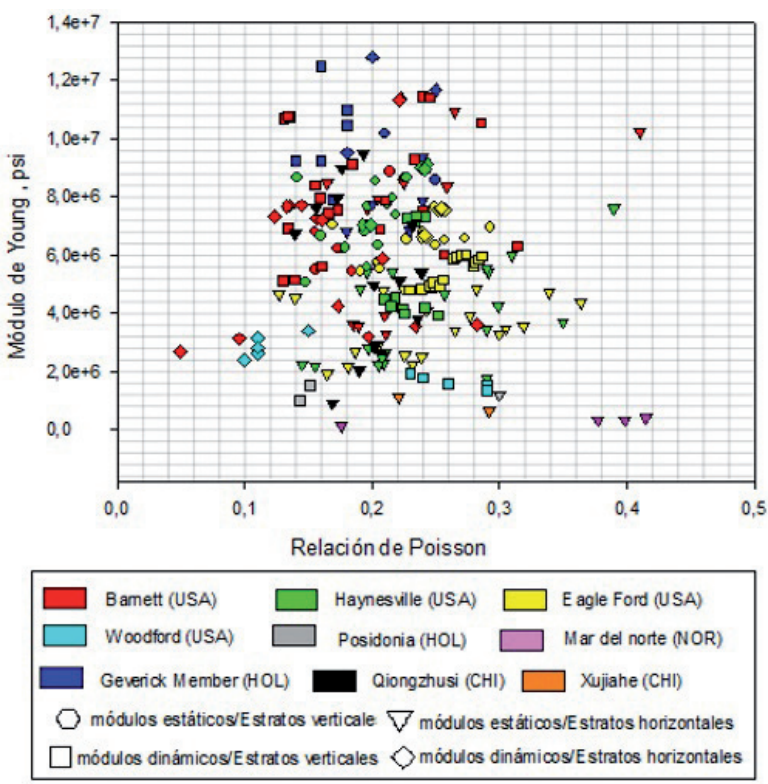

Figura 1. Módulos elásticos para diferentes formaciones de shale gas a nivel mundial.

\section{MINERALOGÍA EN FORMACIONES DE SHALE GAS A NIVEL MUNDIAL}

La descripción mineralógica es una parte esencial en la caracterización mecánica de yacimientos no convencionales, en especial la cuantificación del contenido de arcillas tomado como la fracción dúctil y el contenido de Cuarzo y carbonatos como la fracción frágil de la roca [34]. Una formación de shale gas prospecta a fracturar debe contener menos de un $40 \%$ en peso de minerales arcillosos, los cuales afectan negativamente su fragilidad [4].

Como se muestra en la Figura 2, en Estados Unidos (USA) se ha estudiado ampliamente la composición mineralógica de algunas de las formaciones de shale gas más importantes del mundo como lo son Barnett, Marcellus, Haynesville, Eagle Ford y Woodford [1][7] [17][18][21][22][25][27][33][35][37].

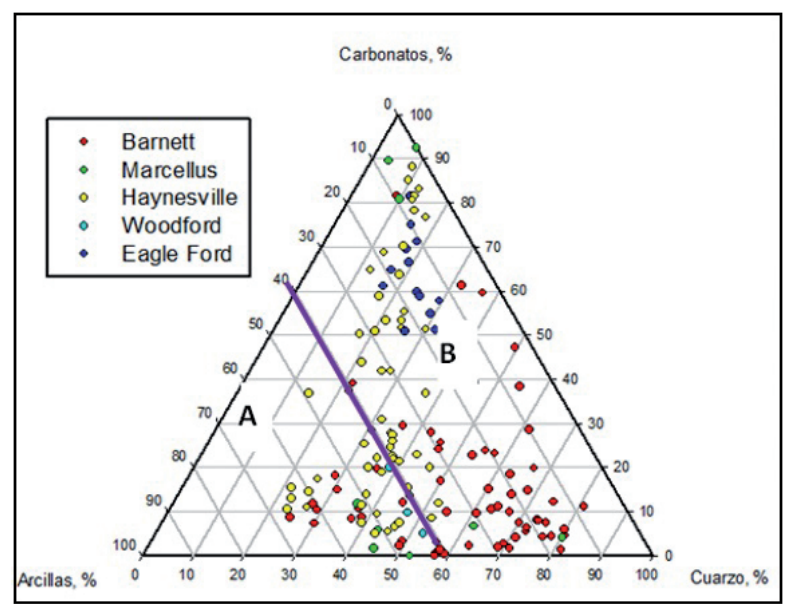

Figura 2. Mineralogía en shales de Estados Unidos clasificados en zona dúctil (A) y zona frágil (B).

De igual manera la Figura 3 ilustra como en Europa nororiental, específicamente en países como Holanda, Dinamarca, Suecia, y Noruega, se han realizado estudios donde involucran la mineralogía de algunas de las formaciones de shale gas más importantes del continente [3][4][23][24][26][30].

Asimismo la Figura 4 muestra como en China (CHI), país con las mayores reservas de shale gas en el mundo [2], se ha publicado gran cantidad de información referente a la composición mineralógica de las formaciones de shale gas con mayor prospección de la zona [5][8][11][12][27][36][39]. 


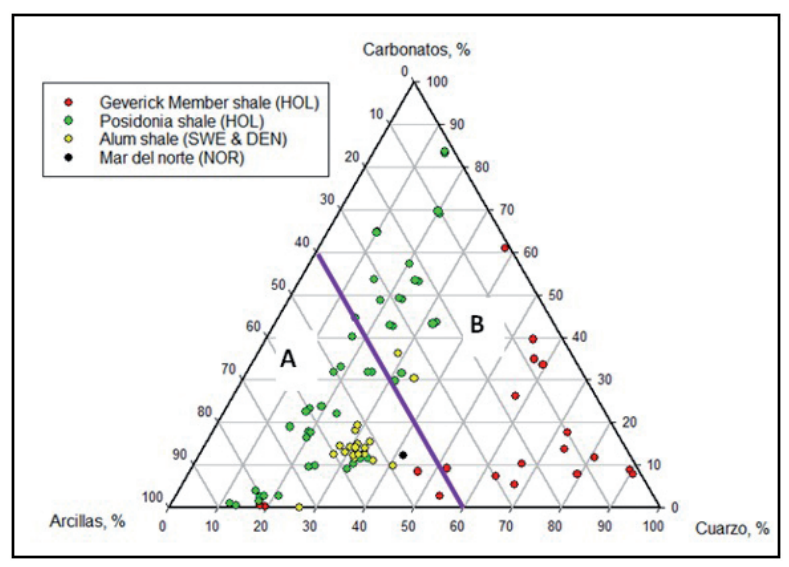

Figura 3. Mineralogía en shales de Europa nororiental clasificados en zona dúctil (A) y zona frágil (B).

Fuente: Autores.

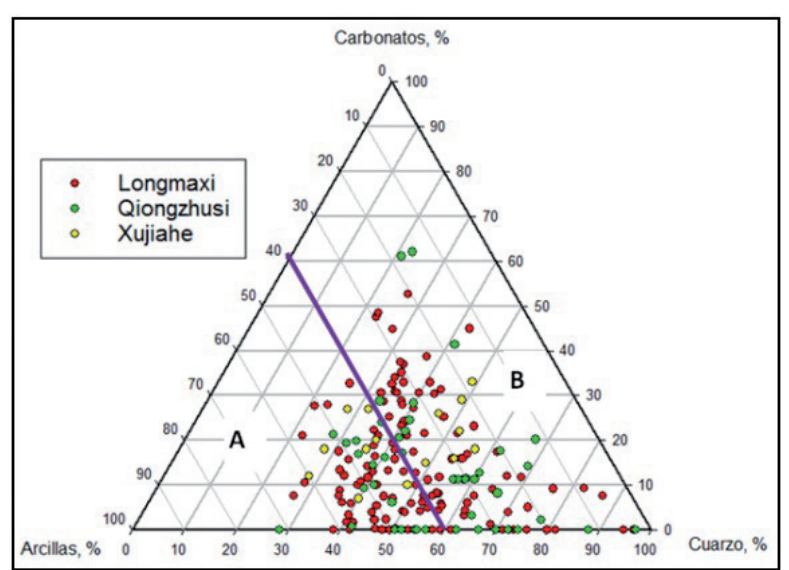

Figura 4. Mineralogía en shales de China clasificados en zona dúctil (A) y zona frágil (B).

\section{INDICE DE FRAGILIDAD A PARTIR DE MÓDULOS ELASTICOS Y MINERALOGÍA}

El índice de fragilidad (BI) es un parámetro expresado en porcentaje, que indica que tan frágil es la roca, i.e. con que facilidad se van a desarrollar las fracturas ante un eventual fallamiento. Dicho parámetro se puede expresar en función del módulo de Young y la relación de Poisson como módulos estáticos [32] (ecuaciones $3,4,5)$ o como módulos dinámicos derivados de datos de inversión sísmica [14] (ecuaciones 6 y 7). A mayor módulo de Young la roca se deforma menos bajo una carga de esfuerzo, por lo tanto es más propensa a fallar; a menor relación de Poisson la roca sufre una menor extensión lateral, por lo tanto será menos flexible y más propensa a fallar bajo esfuerzos.

$$
\begin{gathered}
E_{B R I T}=\left|\frac{E S-E \min }{E \max -E \min }\right| * 100 \\
v_{B R I T}=\left|\frac{V S-V \max }{V \operatorname{Vmin}-V \max }\right| * 100 \\
B I=\left|\frac{E_{B R I T}+v_{B R I T}}{2}\right|
\end{gathered}
$$

Donde:

$E_{B R I T}=$ Fragilidad con base en el módulo de Young [\%] $E S=$ Módulo de Young medido [psi]

Emin $=1 \mathrm{E}+06$ [psi], más elástico.

$E \max =10 \mathrm{E}+06$ [psi], más frágil.

$v_{B R I T}=$ Fragilidad con base en la relación de Poisson [\%]

$v$ SRelación de Poisson medida. [adimensional]

$v \min =0.05$ [adimensional], más frágil.

$v \max =0.4$ [adimensional], más elástico.

$B I=$ Índice de fragilidad $[\%]$

En caso de no contar con mediciones estáticas, los términos de ES y S pueden ser sustituidos en la ecuación 3 por las ecuaciones 6 y 7 , respectivamente:

$$
\begin{gathered}
E^{\prime}=\frac{\rho V_{S}^{2}\left(3 V_{p}^{2}-4 V_{S}^{2}\right)}{\left(V_{p}^{2}-V_{S}^{2}\right)} * 1,4503 * 10^{-4} \\
v^{\prime}=\frac{\left(V_{p}^{2}-2 V_{S}^{2}\right)}{2\left(V_{p}^{2}-V_{S}^{2}\right)}
\end{gathered}
$$

Donde:

$\rho=$ Densidad promedio de la roca $\left[\mathrm{g} / \mathrm{cm}^{3}\right]$

$V_{p}=$ Velocidad de onda compresional $[\mathrm{Km} / \mathrm{s}]$

$V_{s}^{p}=$ Velocidad de onda de corte $[\mathrm{Km} / \mathrm{s}]$

$E^{\prime}=$ Módulo de Young dinámico [psi]

$v^{\prime}=$ Relación de Poisson dinámica [psi]

En el mismo orden de ideas se puede calcular el índice de fragilidad a partir de la composición mineralógica de la muestra de shale [16] (ecuación 8). Como se mencionó anteriormente, el contenido de Cuarzo representa la porción frágil de la muestra y las arcillas la porción dúctil.

$$
B I=\frac{Q}{Q+C+C l} * 100
$$

Donde:

$Q=$ Contenido de Cuarzo [\%]

$C=$ Contenido de Carbonatos $[\%]$ 
$C l=$ Contenido de arcillas $[\%]$

$B I=$ Índice de fragilidad [\%]

\section{CÁLCULO DEL INDICE DE FRAGILIDAD EN FORMACIONES DE SHALE GAS A NIVEL MUNDIAL}

Un índice de fragilidad mayor o igual a 40\% representa una formación prospecta a fracturar hidráulicamente [31]. Sin embargo, valores menores a $40 \%$ pueden

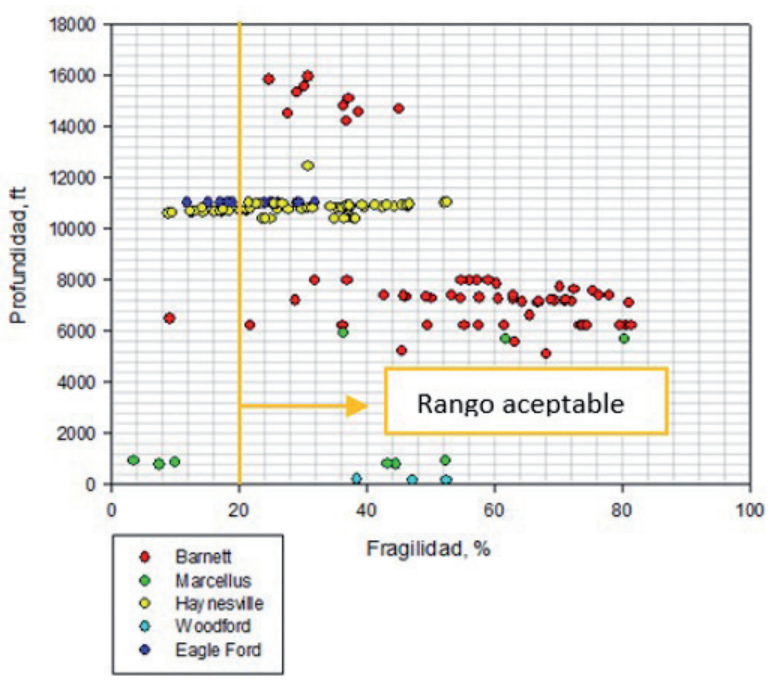

Figura 5. Fragilidad en función de la mineralogía en formaciones de shale gas en USA. considerarse aceptables en intervalos de interés o "sweet spots" teniendo en cuenta el fluido de fractura apropiado a utilizar.

Las Figuras 5, 6 y 7 ilustran los valores obtenidos en el cálculo del índice de fragilidad en función de la mineralogía para USA, Europa y China. A pesar de que la gran mayoría de las formaciones presentan un alto grado de heterogeneidad, los valores de índice de fragilidad se encuentran en el rango aceptable (mayor a $20 \%$ ).

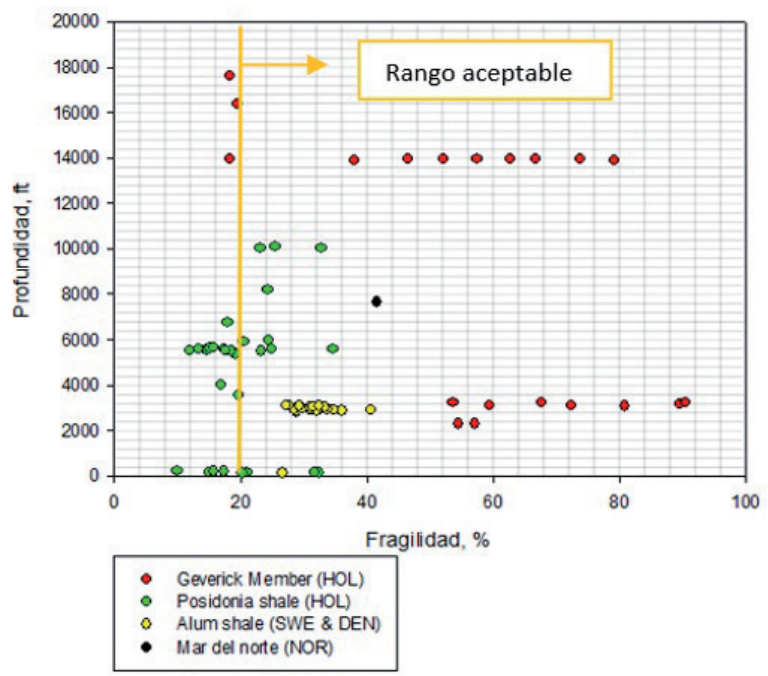

Figura 6. Fragilidad en función de la mineralogía en formaciones de shale gas en Europa.

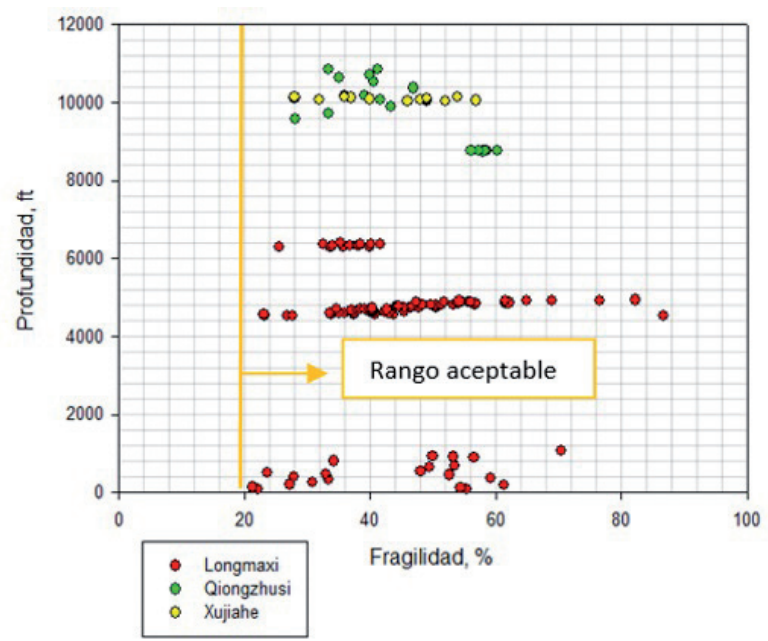

Figura 7. Fragilidad en función de la mineralogía en formaciones de shale gas en China. 
Las Figuras 8, 9 y 10 muestran los valores obtenidos en el cálculo del índice de fragilidad a partir de módulos elásticos (y) estáticos y dinámicos en muestras de diferentes formaciones de shale gas a nivel mundial. Allí se presenta un rango de valores de fragilidad más

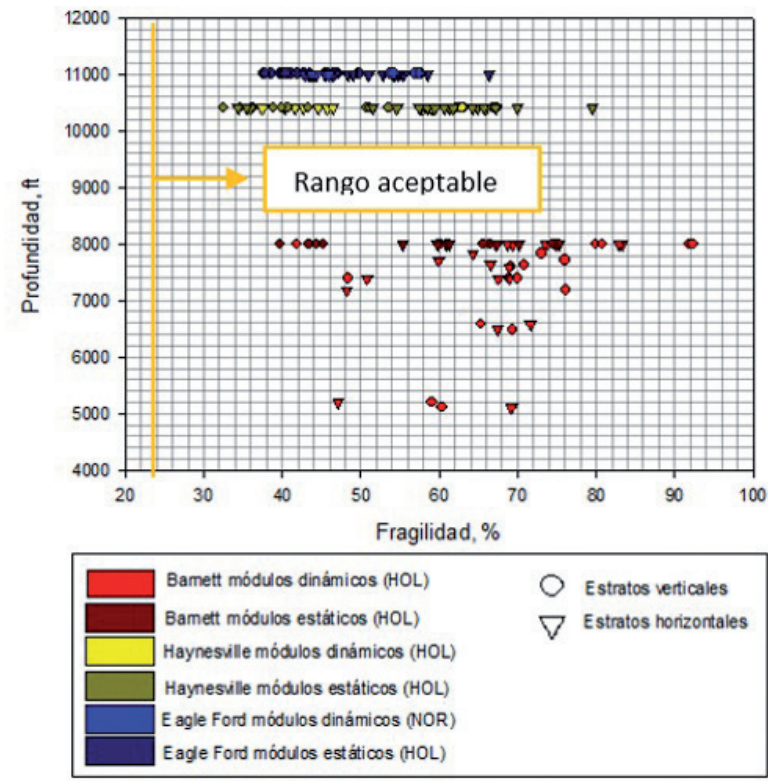

Figura 8. Fragilidad de muestras en función de módulos elásticos estáticos y dinámicos en formaciones de shale gas en USA. optimistas que los mostrados analizando la mineralogía, teniendo en cuenta que los módulos elásticos dinámicos casi siempre son mayores a los módulos elásticos estáticos lo que implica rocas aparentemente más frágiles.

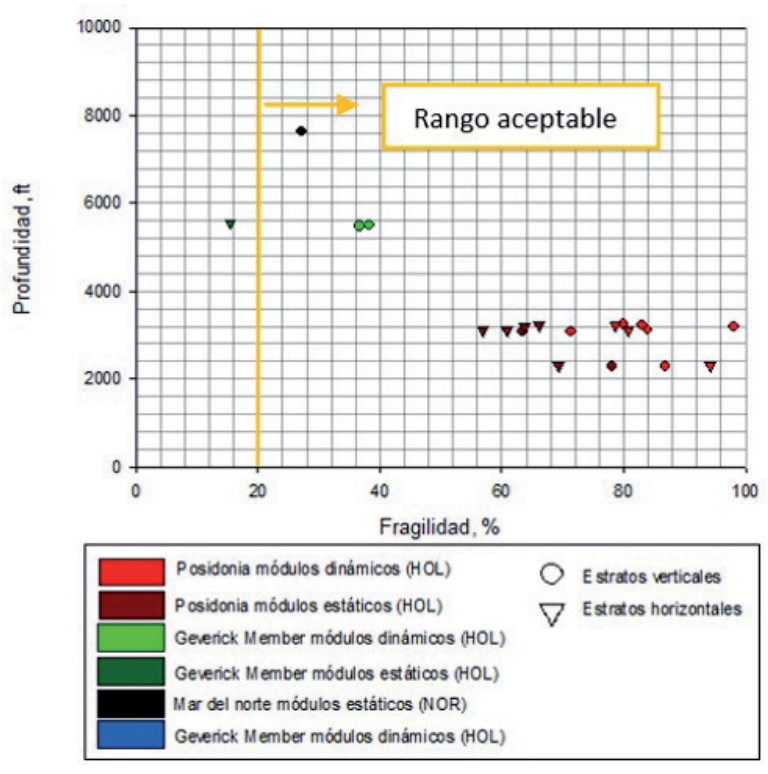

Figura 9. Fragilidad de muestras en función de módulos elásticos estáticos y dinámicos en formaciones de shale gas en Europa.

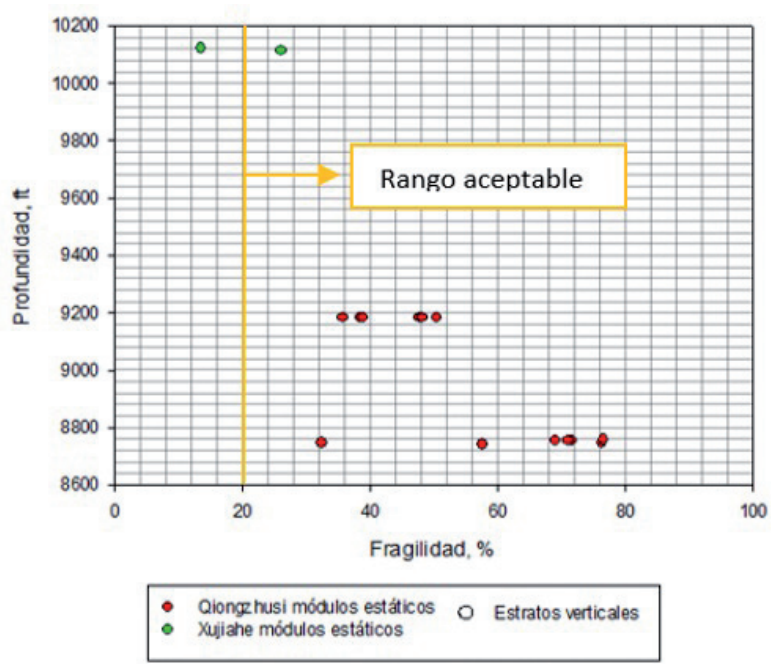

Figura 10. Fragilidad de muestras en función de módulos elásticos estáticos y dinámicos en formaciones de shale gas en China. 
De acuerdo a los cálculos realizados, se identificó que las formaciones con altos contenidos de cuarzo (e.g. Barnett, Qiongzhusi, Posidonia) presentaban valores de fragilidad similares en cuanto a los dos métodos utilizados para el respectivo cálculo, por lo tanto estas formaciones fueron las escogidas para identificar los posibles intervalos de interés que son técnicamente fracturables. Para definir los sweet spots más representativos, se recolectaron datos de TOC para cada formación a determinadas profundidades y se escogieron aquellos con valores de TOC superiores a $2 \%$ en peso ( $\% \mathrm{wt})$, los cuales se muestran en la Tabla 3 .

Tabla 3. Intervalos de interés definidos bajo parámetros de fragilidad y TOC.

\begin{tabular}{|cccc|}
\hline Formación & Sweet spot, ft & $\begin{array}{c}\text { Fragilidad } \\
\text { promedio } \\
\text { \% }\end{array}$ & $\begin{array}{c}\text { TOC } \\
\text { promedio, } \\
\text { wt\% } \%\end{array}$ \\
\hline Barnett & $7180-7390$ & 61.6 & $3.77[9]$ \\
\hline Marcellus & $5694-5700$ & 71.05 & $4.57[6]$ \\
\hline G.Member & $13933-13950$ & 58 & $5.51[24]$ \\
\hline Posidonia & $5485-5555$ & 25.5 & $2.27[24]$ \\
\hline Qiongzhusi & $8740-8760$ & 67.1 & $2.35[19]$ \\
\hline
\end{tabular}

Finalmente se determinó el fluido de fracturamiento óptimo para cada intervalo de interés teniendo en cuenta la fragilidad promedio de cada formación (Tabla 4). Se debe disponer de grandes cantidades de fluido de baja viscosidad para fracturar formaciones frágiles i.e. índices de fragilidad mayores a $50 \%$. Por otro lado, es necesario un fluido de alta viscosidad que pueda sostener una concentración alta de propante para efectuar y asegurar la integridad de la fractura en formaciones con comportamiento dúctil i.e. índices de fragilidad menores a $20 \%$ [20][38][39].

Tabla 4. Fluido de fractura óptimo para cada intervalo de interés con base en su fragilidad promedio.

\begin{tabular}{|cccc|}
\hline Formación & Sweet spot, ft & $\begin{array}{c}\text { Fragilidad } \\
\text { promedio } \\
\text { \% }\end{array}$ & $\begin{array}{c}\text { Fluido de } \\
\text { fractura } \\
\text { óptimo }\end{array}$ \\
\hline Barnett & $7180-7390$ & 61.6 & Slickwater \\
\hline Marcellus & $5694-5700$ & 71.05 & Slickwater \\
\hline G.Member & $13933-13950$ & 58 & Híbrido \\
\hline Posidonia & $5485-5555$ & 25.5 & Crosslinked \\
\hline Qiongzhusi & $8740-8760$ & 67.1 & Slickwater \\
\hline
\end{tabular}

Fuente: Autores.

En la Tabla 4 se observan los intervalos de interés definidos bajo parámetros de TOC y fragilidad con base en mineralogía y módulos elásticos. Aunque la mayoría de los intervalos posee un índice de fragilidad promedio mayor a $50 \%$, el intervalo encontrado en la formación Posidonia posee un BI promedio de $25.5 \%$, que representa una formación dúctil la cual no sería viable fracturar. Sin embargo, el análisis del fluido de fractura óptimo a utilizar junto a la consideración de un alto TOC presente en la formación, hace de este intervalo un sweet spot prospecto.

\section{CONCLUSIONES}

La relación directa entre el contenido de Cuarzo y el módulo de Young permite identificar zonas frágiles a fracturar en formaciones de shale gas.

Contenidos de arcilla mayores a un $40 \%$ en formaciones de shale gas tienen una relación directa con un aumento drástico en la relación de Poisson, lo cual requiere de la selección de fluidos de fractura óptimos para estimular sweet spots con fragilidades menores a un $40 \%$.

Valores de TOC mayores a $2 \%$ wt en intervalos de interés, implican que a una determinada profundidad la formación puede generar grandes cantidades de hidrocarburos lo cual contribuye a la viabilidad técnica del fracturamiento.

En Colombia, el análisis integrado de la mineralogía, el TOC y los módulos elásticos en formaciones de shale permitiría identificar nuevos prospectos de producción, al igual que se podrían definir las profundidades ideales para fracturar hidráulicamente formaciones de shale gas conocidas.

\section{AGRADECIMIENTOS}

Grupo de Investigación de Estabilidad de Pozo GIEPUIS, ICP-Ecopetrol.

\section{BIBLIOGRAFÍA}

1. Abousleiman, Y., Tran, M., Hoang, S., Bobko, C., Ortega, A., Ulm, F. Geomechanics field and laboratory characterization of Woodford shale: the next gas play. SPE 110120. 2007.

2. Administration, U.S Energy Information. Technically Recoverable Shale Oil and Shale Gas Resources. Washington : s.n. USA 2013.

3. Bignonnet, F. Caracterisation experimentale et modelisation micro-mecanique de la permeabilite et la resistance de roches agileuses. Thèse de doctorat, Universitè Paris-Est. France. 2014. 
4. Bouw, S., Lutgert, J. Shale plays in the Netherlands. SPE 152644. 2012.

5. Caineng, Z., Dazhong, D., Shejiao, W., Jianzhong, L., Xinjing, L., Yuman, W., Denghua, L., Keming, C. Geological characteristics and resource potential of shale gas in China. Petroleum Exploration and Development Journal. Vol. 37, pp 641 - 6542010.

6. Call, T. Geomechanical properties of Marcellus shale core samples within a sequence stratigraphic framework. Master thesis, Pennsylvania State University. USA. 2012.

7. Chen, D. Microstructure study on Barnett shale. Master Thesis, University of Houston. USA. 2012.

8. Chenji, W., Guan, Q., Wei, G., Bicheng, Y., Killough, J., Hongyan, W., Honglin, L. Characterization and analysis on petrophysical parameters of a marine shale Gas Reservoir. SPE 165380. 2013.

9. Cronin, M.B. Core-scale heterogeneity and dualpermeability pore structure in the Barnett Shale. Master thesis, University of Texas at Austin. USA. 2014.

10. Dooley, J. Macroscopic physics online text. Millersville University. 2010. http://www. millersville.edu/ jdooley/macro/derive/elas 1/ poissn/poissn.htm

11. Feng, Y., Kai, W., Blanton, E., Winston, Z. Case study: a challenging large-scale fracturing in Sichuan basin. IPTC 18104. 2014.

12. Feng, Y., Zhengfu, N., Qing, W., Huiqing, L., Shidong, Z., Hongmei, L. Integrated study of reservoir characteristics of a shale gas reservoir: a case study from Sichuan basin of China. SPE 165870. 2013.

13. Glorioso, J., Rattia, A. Unconventional reservoirs: basic petrophysical concepts for shale gas. SPE 153004. 2012.

14. Gray, D., Anderson, P., Logel, J., Delbecq, F., Schmid, R. Estimation of stress and Geomechanical properties using 3D seismic data. EAGE, first break. Vol. 30. March 2012.

15. Howard, D., Angus, J.Acoustics and Psychoacoustics, ISBN 0240519957. Third edition. 2006.
16. Jarvie, D.M., Hill, R.J., Ruble, T.E., Pollastro, R.M. unconventional Shale-gas Systems: the Mississippian Barnett shale of North Central Texas, as one model for thermogenic shale-gas assessment. AAPG Bulletin. Vol. 9, pp 475-499.2007

17. Jiang, T. Connection of elastic and transport properties: effective medium study in anisotropic porous media. Doctorate thesis , University of Houston. USA. 2013.

18. Kinley, T.J. Geology and hydrocarbon potential of the Barnett shale (Mississippian) in the northern Delaware basin, west Texas and southeastern New Mexico. Master thesis, Texas Christian University. USA. 2006.

19. Lau, H., Yu, M. Production technology challenges of tight and shale gas production in China. IPTC 17096. 2013.

20. Li, Q., Chen, M., Jin, Y., Zhou, Y., Wang, F., Zhang, R. Rock mechanical properties of shale gas reservoir and shale gas reservoir and their influences on hydraulic fracture. IPTC 16580. 2013.

21.Li, Y., Ghassemi, A. Creep behavior of Barnett, Haynesville, and Marcellus shale. ARMA12-330. 2012.

22. Mullen, J. Petrophysical characterization of the Eagle Ford shale in south Texas. SPE 138145. 2010.

23. Muniz, E., Fontoura, S. Cu triaxial tests on North Sea shale. ARMA/USRMS 05-814. 2005.

24.Noordoven, Q.A.L.V. Characterization of production potential in Jurassic and Carboniferous shale plays of the Netherlands. Master thesis, Delft University of technology, Holland. 2011.

25. Pollard, D.D. and P. Segall, Theoretical displacements and stresses near fractures in rock; with applications to faults, joints, veins, dikes, and solution surfaces. Fracture mechanics of rock, ed. B.K. Atkinson. London, United Kingdom (GBR): Acad. Press, London. England. 1987.

26. Pool, W., Geluk, M., Abels, J., Tiley, G., Idiz, E., Leenaarts, E. Assessment of an unusual European shale gas play: the Cambro-Ordovician Alum shale, southern Sweden. Paper SPE 152339. 2012. 
27. Qionghui, L., Chen, M., Jin, Y., Wang, F. Experimental research on failure modes and mechanical behaviors of gas-bearing shale. Chinese Journal of Rock Mechanics and Engineering, Vol 31, pp 3764-3770.2012.

28. Rabe, C. \& Cherrez, J. Laboratory characterization of Norwegian North Sea shale. ISRM 2008-052. 2008.

29. Ravestein, T. Fraccability determination of a Posidonia Shale Formation analogue through geomechanical experiments and micro-CT fracture propagation analysis. Master thesis, Delft University of Technology, Holland. 2014.

30. Rexer, T. Nanopore characterisation and gas sorption potential of European gas shales. Doctorate thesis, Newcastle University, England. 2014.

31. Rickman, R., Mullen, M., Petre, E., Grieser, B., Kundert, D. A practical use of shale petrophysics for stimulation design optimization: All shale plays are not clones of the Barnett shale. SPE 115258. 2008.

32. Rickman, R., Mullen, M., Petre, E., Grieser, B., Kundert, D. Petrophysics key in stimulating shales. The American Oil \& Gas Reporter. March, 2009.

33. Shukla, P., Kumar, V., Curtis, M., Sondergeld, C. H., Rai, C. S. Nanoindentation studies on shales. University of Oklahoma. ARMA 2013-578. USA. 2013.
34. Sone, H, Zoback, M. Mechanical properties of shale-gas reservoir rocks - part 1: static and dynamic elastic properties and anisotropy. Geophysics Journal. Vol. 78, pp D381 - D392. 2013.

35. Sone, H. Mechanical properties of shale gas reservoir rocks and its relation to the in-situ stress variation observed in shale gas reservoirs. Doctorate thesis, Stanford University. USA. 2012.

36. Tan, J. Shale gas potential of the major marine shale formations in the upper Yangtze platform, south China. Doctorate thesis, Technische Universität Berlin. Germany. 2014.

37. Tepper, B., Baechle, G., Keller, J., Walsh, R., Quint, E. Petrophysical evaluation of shale oil \& gas opportunities in emerging shale plays; some examples and learnings from the Americas. IPTC 16926. 2013.

38. Yuan, F., Wang, K., Balnton, E., Zeng, W. Case study: A challenging large-Scale fracturing in Sichuan basin. IPTC 18104. 2014.

39. Zonggang, Lv., Wang, L., Stufen, D., Chong, K., Wooley, J., Qiang, W., Peng, J. China's marine Qiongzhusi shale play: first deep Asia pacific region horizontal multiple stage frac: case history, operation \& execution. IPTC 16391. 2013.

Recepción: 22 de Julio de 2015

Aceptación: 8 de Septiembre de 2015 